\title{
Finite-size scaling of the quasispecies model
}

\author{
P. R. A. Campos and J. F. Fontanari \\ Instituto de Física de São Carlos \\ Universidade de São Paulo \\ Caixa Postal 369 \\ 13560-970 São Carlos SP \\ Brazil
}

\begin{abstract}
We use finite-size scaling to study the critical behavior of the quasispecies model of molecular evolution in the single-sharp-peak replication landscape. This model exhibits a sharp threshold phenomenon at $Q=Q_{c}=1 / a$, where $Q$ is the probability of exact replication of a molecule of length $L$ and $a$ is the selective advantage of the master string. We investigate the sharpness of the threshold and find that its characteristics persist across a range of $Q$ of order $L^{-1}$ about $Q_{c}$. Furthermore, using the data collapsing method we show that the normalized mean Hamming distance between the master string and the entire population, as well as the properly scaled fluctuations around this mean value, follow universal forms in the critical region.

87.10.+e, 64.60.Cn
\end{abstract}

Typeset using REVTEX 
Although the so-called error threshold phenomenon, which limits the length $L$ of competing self-reproducing molecules, is acknowledged as one of the main outcomes of Eigen's quasispecies model [1],2], the full characterization of the error threshold transition for finite $L$ has not been satisfactorily carried out yet. In fact, similarly to the definition of the critical temperature for finite lattices, there is no generally accepted definition of the term error threshold for finite $L[3]$. Nevertheless, the study of the systematic deviations from the infinite length limit behavior introduced by the finite-size effects, besides being practically independent of the definition adopted, gives valuable information on the behavior of the relevant macroscopic quantities near the critical region [四, 5].

In the quasispecies model, a molecule is represented by a string of $L$ digits $\vec{s}=$ $\left(s_{1}, s_{2}, \ldots, s_{L}\right)$, with the variables $s_{\alpha}$ allowed to take on $\kappa$ different values, each representing a different type of monomer used to build the molecule. For sake of simplicity, in this work we will consider only binary strings, i.e., $s_{\alpha}=0,1$. The concentrations $x_{i}$ of molecules of type $i=1,2, \ldots, 2^{L}$ evolve in time according to the following differential equations [1, 2]

$$
\frac{d x_{i}}{d t}=\sum_{j} W_{i j} x_{j}-\left[D_{i}+\Phi(t)\right] x_{i},
$$

where the constants $D_{i}$ stand for the death probability of molecules of type $i$, and $\Phi(t)$ is a dilution flux that keeps the total concentration constant. This flux introduces a nonlinearity in (1), and is determined by the condition $\sum_{i} d x_{i} / d t=0$. More pointedly, assuming $D_{i}=0$ for all $i$ and $\sum_{i} x_{i}=1$ yields

$$
\Phi=\sum_{i, j} W_{i j} x_{j}
$$

The elements of the replication matrix $\mathbf{W}$ are given by

$$
W_{i i}=A_{i} q^{L}
$$

and

$$
W_{i j}=A_{i} q^{L-d(i, j)}(1-q)^{d(i, j)} \quad i \neq j
$$


where $A_{i}$ is the replication rate or fitness of molecules of type $i$, and $d(i, j)$ is the Hamming distance between strings $i$ and $j$. Here $0 \leq q \leq 1$ is the single-digit replication accuracy, which is assumed to be the same for all digits.

In this work we will consider the simplest and probably most studied replication landscape, namely the single-sharp-peak replication landscape, in which we ascribe the replication rate $a>1$ to the so-called master string $(0,0, \ldots, 0)$, and the replication rate 1 to the remaining strings. In this context, the parameter $a$ is termed selective advantage of the master string. As the replication accuracy $q$ decreases, two distinct regimes are observed in the population composition: the quasispecies regime characterized by the master string and its close neighbors, and the uniform regime where the $2^{L}$ strings appear in the same proportion. The transition between these regimes occurs at the error threshold $q_{c}$. To study this transition for large $L$ it is more convenient to introduce the probability of exact replication of an entire string, namely

$$
Q=q^{L}
$$

so that for $L \rightarrow \infty$ the transition occurs at [1],2]

$$
Q_{c}=\frac{1}{a}
$$

Although there is a consensus that a thermodynamic order-disorder phase transition occurs in the limit $L \rightarrow \infty$ only [6 \&], there is some disagreement on the order of the transition. On the one hand, the mapping of the steady-state solution of the chemical kinetic equations (1) into the surface properties of a semi-infinite two-dimensional lattice system in thermodynamic equilibrium indicates that the relevant order parameter, namely, the mean normalized Hamming distance $d$ between the master string and the entire population, vanishes continuously at $Q_{c}[7]$. However, due to the enormous difficulty of solving the self-consistent equations that describe the equilibrium surface properties that analysis was restricted to $L=20$ [7]. On the other hand, a thorough investigation of an alternative mapping of equations (1) into a problem of directed polymers in a random medium indicates 
that the concentration of master strings presents a discontinuity at $Q=Q_{c}$ [8]. Since this mapping allows for the exact solution of the quasispecies model in the single-sharp-peak replication landscape for generic lengths $L$, that result implies that the transition for $L \rightarrow \infty$ is definitely of first order [8].

The aim of this work is to investigate the finite-size effects near the error threshold transition. Of particular interest is the determination of the sharpness of the threshold, namely, the range of $Q$ about $Q_{c}$ where the threshold characteristics persist. As we expect that the size of this region shrinks to zero like $L^{-1 / \nu}$ as $L \rightarrow \infty$, our goal is to estimate the value of the exponent $\nu \geq 0$ using finite-size scaling or, more precisely, the data collapsing method [5]. Our approach is in the same spirit of the finite-size scaling of combinatoric problems [9], for which there is no geometric criterion for defining a quantity analogous to the correlation length $\xi$, and so the success of the method in accounting for the size dependence of the order parameters cannot be attributed to the divergence of $\xi$ and the consequent onset of a second order phase transition. In fact, instead of attempting to map the chemical kinetic equations (11) into a equilibrium statistical mechanics problem, we resort to a simpler and more direct approach, namely, the exact numerical solution of those equations in the steady-state regime for molecule lengths up to $L=150$.

As pointed out by Swetina and Schuster [10], for the single-sharp-peak replication landscape the $2^{L}$ molecular concentrations $x_{i}$ can be grouped into $L+1$ distinct classes according to their Hamming distances to the master string. This procedure allows the description of the chemical kinetics by the following $L+1$ coupled first-order differential equations [10]

$$
\frac{d Y_{P}}{d t}=\sum_{R=0}^{L} M_{P R} Y_{R}+(a-1) Y_{0} M_{P 0}-Y_{P}\left[1+Y_{0}(a-1)\right],
$$

where $Y_{P}$ denotes the concentrations of molecules in class $P=0, \ldots, L$. Clearly, $\sum_{P} Y_{P}=1$. Here $M_{P R}$ stands for the probability of mutation from a molecule of type $R$ to a molecule of type $P$ and is given by

$$
M_{P R}=\sum_{I=I_{l}}^{I_{u}}\left(\begin{array}{c}
R \\
I
\end{array}\right)\left(\begin{array}{c}
L-R \\
P-I
\end{array}\right) q^{L-P-R+2 I}(1-q)^{P+R-2 I},
$$


where $I_{l}=\max (0, P+R-L)$ and $I_{u}=\min (P, R)$.

The procedure to obtain the steady-state solution $d Y_{P} / d t=0$ of Eqs. (7) is straightforward. The steady-state concentrations $Y_{P}$ for $P=0, \ldots, L$ can be easily found by solving by iterations the following set of equations

$$
Y_{P}=\frac{\sum_{R=0}^{L} M_{P R} Y_{R}+(a-1) Y_{0} M_{P 0}}{1+Y_{0}(a-1)}
$$

Interestingly, the iteration of these equations is identical to the dynamics of a recently proposed population genetics model based on the neglect of the linkage disequilibrium at the population level [11].

The relevant quantities to describe the structure of the population are the normalized mean Hamming distance between the master string and the whole population, defined by

$$
d=\frac{1}{L} \sum_{P=0}^{L} P Y_{P}
$$

and the average of the squared deviations around $d$,

$$
\sigma^{2}=L^{2} \sum_{P=0}^{L}\left(\frac{P}{L}-d\right)^{2} Y_{P} .
$$

Clearly, $d$ and $\sigma^{2}$ are the analogous to the magnetization and susceptibility in a system of Ising spins. In Figs. 1 and 2 we present $d$ and $\sigma^{2}$, respectively, as functions of the properly normalized probability of exact replication $Q / Q_{c}$. As expected, the results of Fig. 11 show the sharpening of the transition with increasing $L$. Furthermore, all curves intersect at an unique point, namely, the critical point $Q=Q_{c}$. This somewhat unexpected result has proved very useful to locate the threshold in the case that its location is not known apriori [9]. The curves shown in Fig. 2 indicate that the height of the peak of $\sigma^{2}$, denoted by $\sigma_{\max }^{2}$, increases with increasing $L$ like $L^{\gamma / \nu}$. As illustrated in the inset, the ratio $\gamma / \nu$ is given by the slope of the straight line fitting the data points in a plot of $\ln \sigma_{\max }^{2}$ versus $\ln L$. The result $\gamma / \nu=1.96$ is in good agreement with the analytical prediction that the rms amplitude of a quasispecies around the master string $\left(\sqrt{\sigma^{2}}\right)$ is found to diverge algebrically with the exponent 1 as $Q \rightarrow Q_{c}$ [8]. 
The exponent $1 / \nu$ is estimated using the standard data collapsing method [5] as illustrated in Figs. 3 and 4 , where we plot $d$ and $L^{-\gamma / \nu} \sigma^{2}$, respectively, versus $L^{1 / \nu} \epsilon$. Here $\epsilon=\left(Q-Q_{c}\right) / Q_{c}$ is the reduced probability of exact replication. The collapse of the curves for different $L$ was achieved with the exponents $1 / \nu=1$ and $\gamma / \nu=1.958$ regardless of the value of the selective advantage parameter $a$, indicating then the universal character of these exponents. However, as shown in these figures, the universal forms (i.e., scaling functions) followed by the properly scaled order parameters in the critical region depend on $a$. Since $\nu=1$, we note that the characteristics of the threshold transition persist across a range of $Q$ of order $L^{-1}$ about $Q_{c}=1 / a$.

As in the case of combinatoric problems [9], it is surprising that finite-size scaling is so effective to characterize the error threshold transition of the quasispecies model in the single-sharp-peak replication landscape, which is known to be of first order [8]. The collapse of the data for different $L$ into a single, universal curve presented in Figs. 3 and 4 , however, is an incontestable evidence of the usefulness of the finite-size scaling method to investigate threshold phenomena. In fact, the existence of the universal forms presented in those figures together with the characterization of the sharpness of the error threshold are the main results of this paper.

\section{ACKNOWLEDGMENTS}

The work of JFF was supported in part by Conselho Nacional de Desenvolvimento Científico e Tecnológico (CNPq). PRAC holds a FAPESP fellowship. 


\section{REFERENCES}

[1] M. Eigen, Naturwissenchaften 58, 465 (1971).

[2] M. Eigen, J. McCaskill and P. Schuster, Adv. Chem. Phys. 75, 149 (1989).

[3] T. Wiehe, Genet. Res. Camb. 69, 127 (1997).

[4] M. N. Barber, in Phase Transitions and Critical Phenomena, edited by C. Domb and J. L. Lebowitz (Academic Press, London, 1983), Vol. 8.

[5] K. Binder, J. Comp. Phys. 59, 1 (1985).

[6] I. Leuthäusser, J. Chem. Phys. 84, 1884 (1986); J. Stat. Phys. 48, 343 (1987).

[7] P. Tarazona, Phys. Rev. A 45, 6038 (1992).

[8] S. Galluccio, Phys. Rev. E 56, 4526 (1997).

[9] S. Kirkpatrick and B. Selman, Science 264, 1297 (1994).

[10] J. Swetina and P. Schuster, Biophys. Chem. 16, 329 (1982).

[11] D. Alves and J. F. Fontanari, Phys. Rev. E at press. 


\section{FIGURES}

FIG. 1. Normalized mean Hamming distance between the master string and the whole population $d$ as a function of the normalized probability of exact replication $Q / Q_{c}$ for $a=10$, and $L=70$ $(\square), 100(\bigcirc), 120(\triangle)$ and $150(\times)$.

FIG. 2. Standard deviation $\sigma^{2}$ as a function of the normalized probability of exact replication $Q / Q_{c}$. The inset illustrates the procedure used to estimate the ratio $\gamma / \nu$. The parameters and convention are the same as for Fig. 1.

FIG. 3. Normalized mean Hamming distance as a function of the scaled reduced probability of exact replication. The parameters are $1 / \nu=1$ and (from bottom to top) $a=10,20$ and 50 . The convention is the same as for Fig. 1.

FIG. 4. Scaled standard deviation as a function of the scaled reduced probability of exact replication. The parameters are $1 / \nu=1, \gamma / \nu=1.958$, and (from top to bottom at the peak location) $a=10,20$ and 50. The convention is the same as for Fig. 1. 


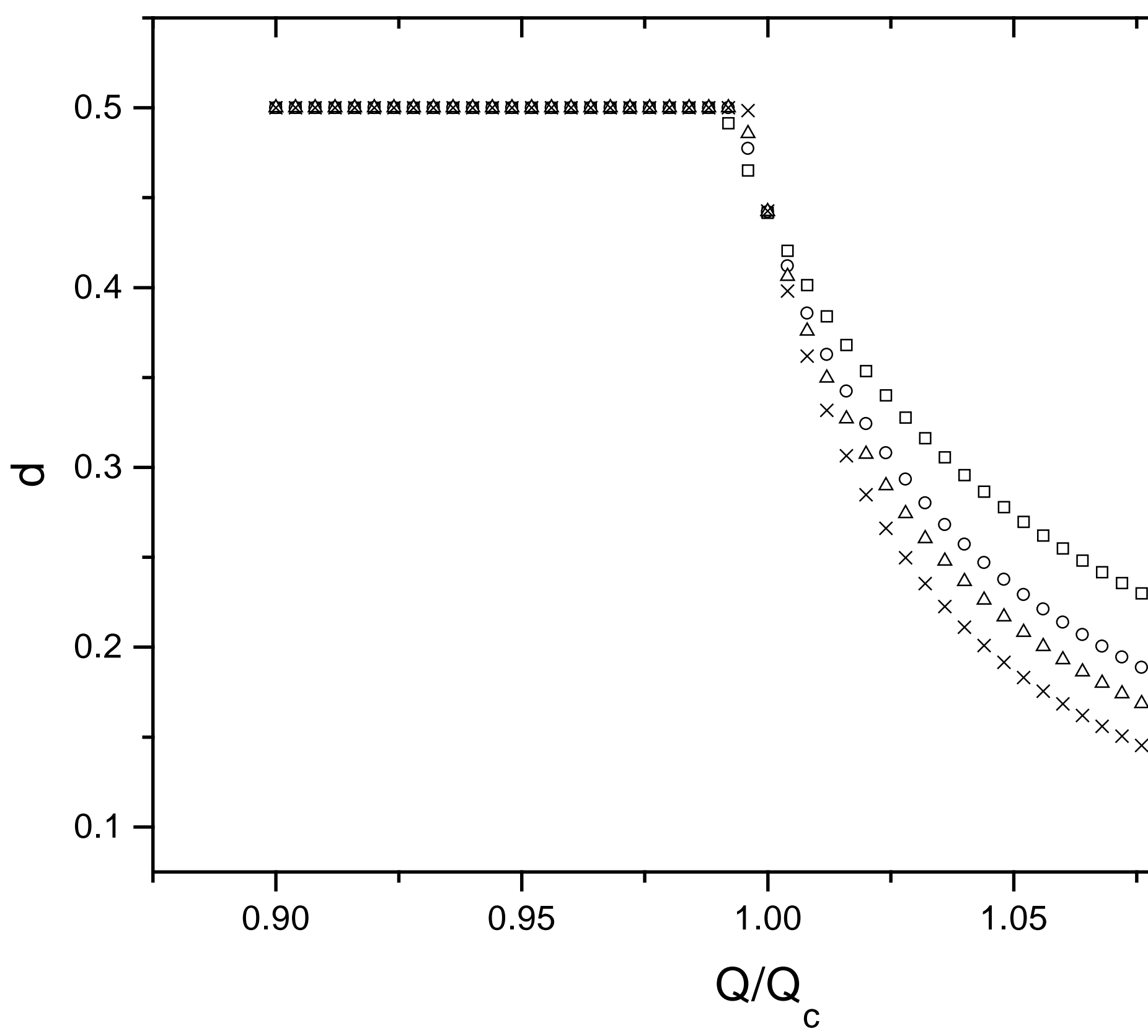




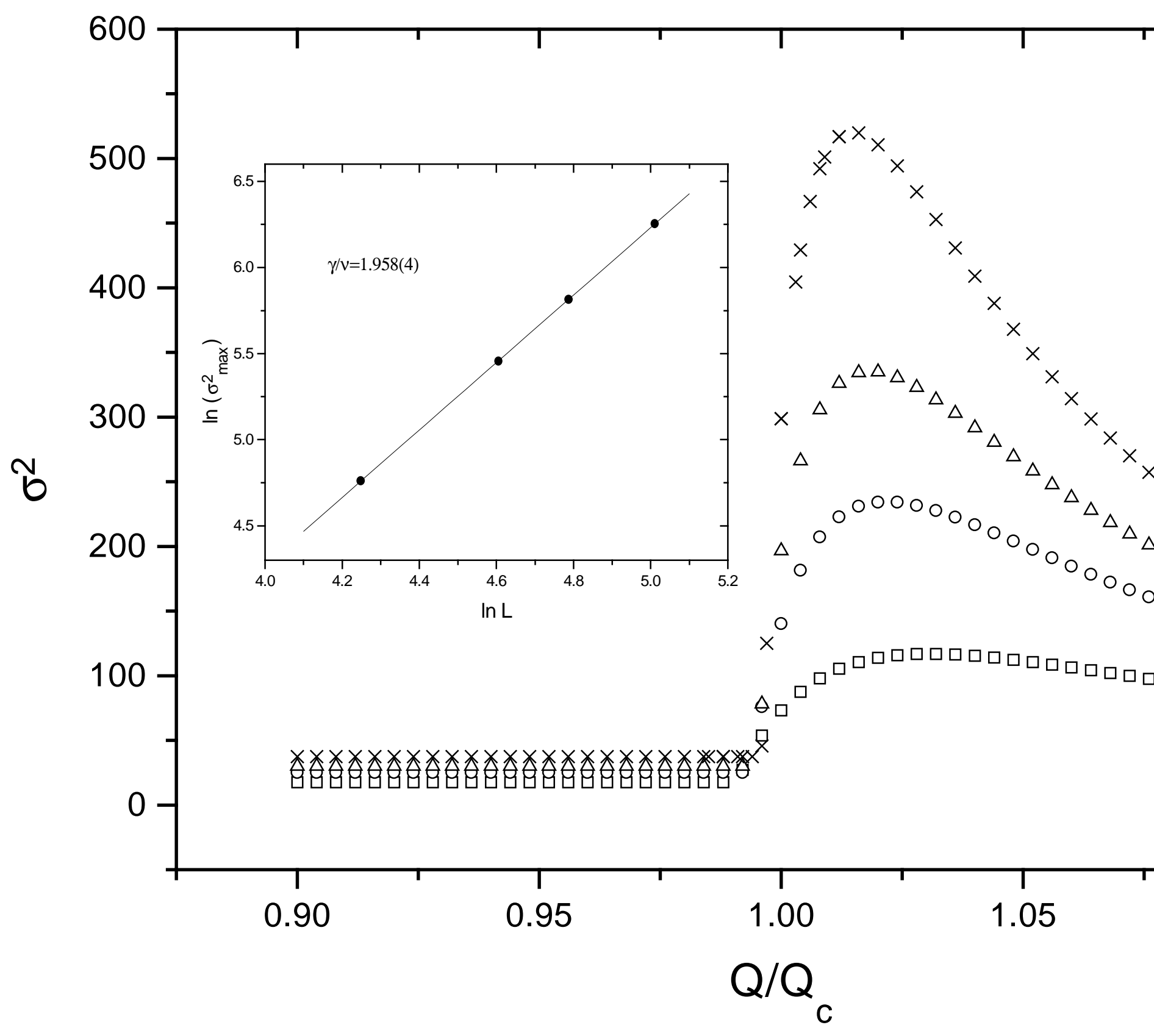




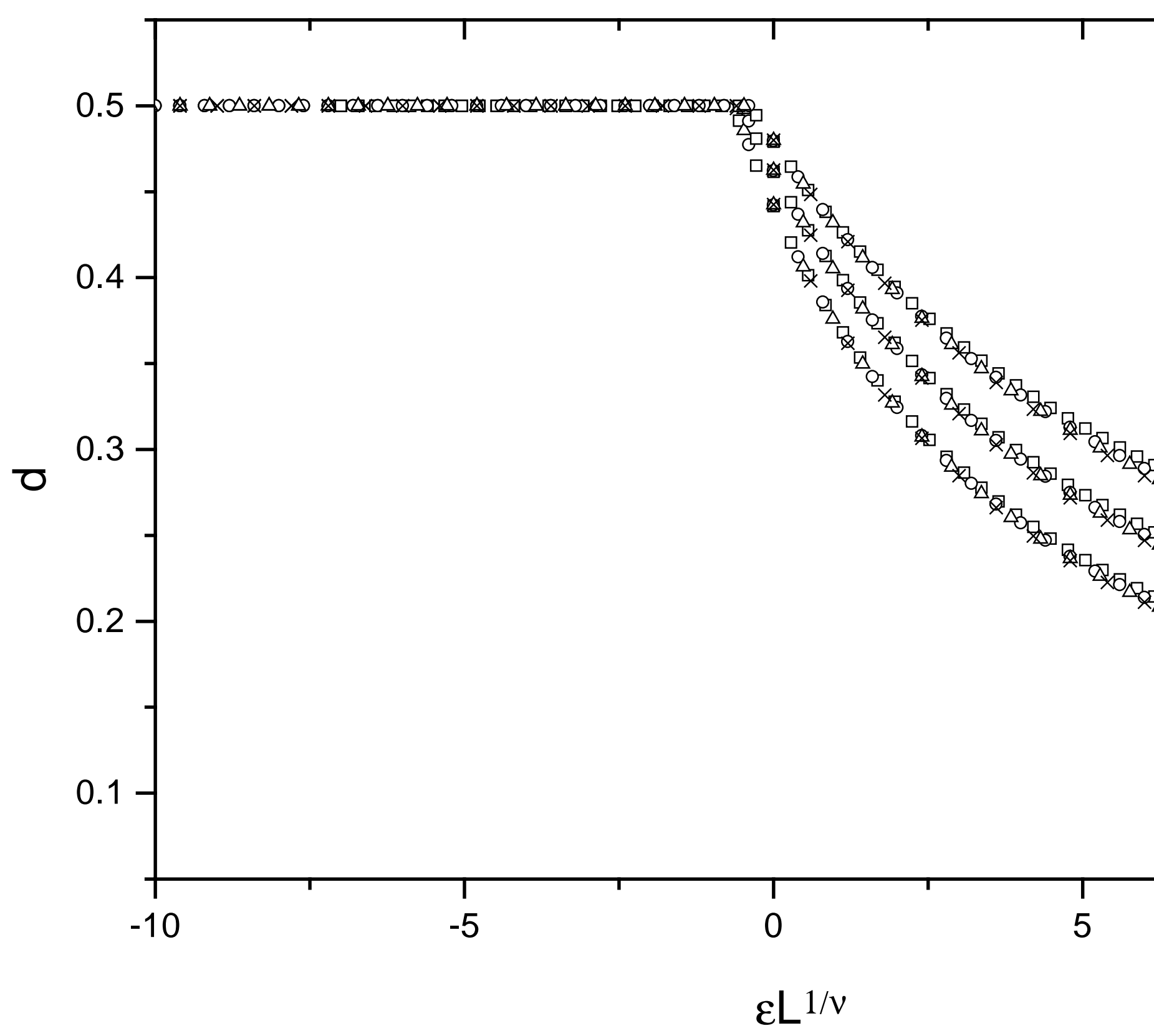




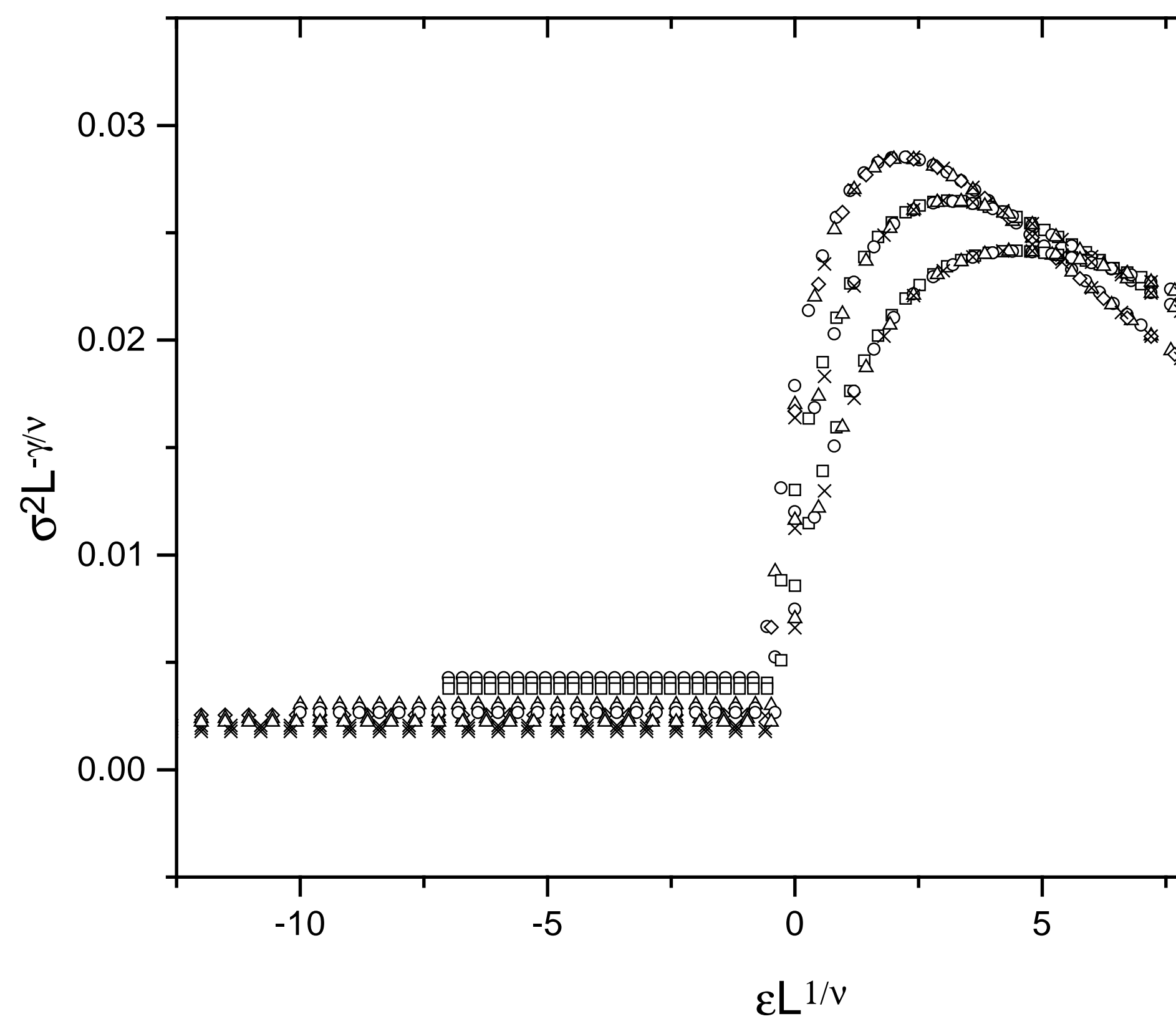

